\title{
Correlation of Morphology of Electrospun Fibers with Rheology of Linear Polyacrylamide Solution
}

\author{
Alexandre A. Vetcher, ${ }^{1, \dagger}$ Ryan Gearheart, ${ }^{1}$ and Victor N. Morozov ${ }^{1,2}$ \\ ${ }^{1}$ The National Center for Biodefense and Infectious Diseases, George Mason University, \\ Manassas, VA 20110, USA \\ ${ }^{2}$ Institute of Theoretical and Experimental Biophysics, Russian Acad. Sci, \\ Pushchino, Moscow region, 142290, Russia
}

(Received March 22, 2007; Accepted April 26, 2007; Published June 12, 2007)

\begin{abstract}
KEY WORDS Electrospinning / Fiber morphology / Linear polyacrylamide / Rheology / Viscosity / [doi:10.1295/polymj.PJ2006269]
\end{abstract}

Nanofibers, fabricated from polymer solutions by electrospinning (ES), have numerous biomedical and technological applications. ${ }^{1-3}$ Much effort has been applied in studying the major parameters affecting fiber diameter and uniformity, ${ }^{1-4}$ one of which is solution viscosity $(\eta) .{ }^{3,4}$ One known method of predicting electrospinnability and estimating nanofiber diameter and quality is based on the De Gennes' scaling concept. ${ }^{5}$ According to it a logarithmic plot of the specific viscosity $\left(\eta_{\mathrm{sp}}=\left(\eta_{\mathrm{s}}-\eta_{0}\right) / \eta_{0}\right.$, where $\eta_{\mathrm{s}}$ and $\eta_{0}$ are zero shear viscosities of solution and solvent respectively) of a polymer solution of specified molecular weight against the concentration, $C$, will contain several areas with different slopes. As the concentration passes from the semidilute unentangled area (with $\eta_{\mathrm{sp}} \sim(C)^{1.25}$ ) to the semidilute entangled area (with $\left.\eta_{\mathrm{sp}} \sim(C)^{4.8}\right)$, a change in slope is observed when the concentration exceeds the entanglement concentration, $C_{e}$. It was also demonstrated that crossing $C_{e}$ results in dramatic changes in electrospun nanofibers as the fibers become free of beads and other defects. ${ }^{6-15}$

The morphology of nanofibers can be characterized by optical microscopy, ${ }^{12,13}$ scanning electron microscopy $(\mathrm{SEM})^{7-10,16}$ and atomic force microscopy (AFM) ${ }^{16,17}$ To reveal certain peculiarities in nanofibers structures a combination of different microscopy approaches are employed. ${ }^{16,18}$

ES of linear polyacrylamide (PAA) is of interest for several reasons. First, the physical properties of polyacrylamide (PAA) solutions in water and other solvents have been well studied. ${ }^{19-22}$ Second, the properties of PAA itself and its numerous modified forms have been studied in cross-linked gels widely used in molecular biology and biotechnology as a separation media. ${ }^{23}$ The multitude of different components introduced into PAA chains upon polymerization ${ }^{24-31}$ makes this material especially attractive for electrospinning. A family of products may be generated with physical and chemical properties modified before, during, or after fiber fabrication.

In most publications the intrinsic viscosity $[\eta]$ of PAA solutions was measured at concentrations which are orders of magnitude lower than those needed to manufacture nanofibers. ${ }^{20,21}$ Moreover, the majority of reported experiments have been performed not with aqueous solutions, but using solvent mixtures and salt additives ${ }^{20,21}$ which potentially affect the ES process. So far no systematic study of the relationship between molecular weight $\left(M_{\mathrm{w}}\right), \eta_{\mathrm{sp}}$, and electrospinnability of PAA has been performed. ${ }^{17}$ In this study we analyze the relationship between specific viscosity and the morphology of electrospun nanofibers for a variety of PAA concentrations and molecular weights.

\section{EXPERIMENTAL}

\section{Materials}

Polyacrylamides (PAA) were obtained from Polysciences, Inc (Warrington, PA) and used without purification. The sample referred to as US was obtained from a 5\% PAA 5MDa solution in water by ultrasound treatment (see Notes to Table I for details).

\section{Viscosity measurements}

Zero shear viscosity was measured with a homemade capillary viscometer ${ }^{32}$ at a temperature of $22^{\circ} \mathrm{C}$ (the same temperature at which ES was performed) provided by a thermostatic room. Polymer solutions were subjected to slow continuous rotation for at least $24 \mathrm{~h}$ to prepare homogeneous solutions. ${ }^{17} \eta_{\mathrm{sp}}$ was calculated according to the formula: 
Table I. Entanglement concentration and other properties of PAA samples

\begin{tabular}{cccc}
\hline $\begin{array}{c}\text { PAA } \\
\text { sample }\end{array}$ & $\begin{array}{c}\text { Average } \\
M_{\mathrm{w}}(\mathrm{kDa}) \text { as } \\
\text { provided by } \\
\text { manufacturer }\end{array}$ & $\begin{array}{c}M_{\mathrm{w}} / M_{\mathrm{n}} \\
\text { as provided by } \\
\text { manufacturer }\end{array}$ & $\begin{array}{c}\text { Entanglement } \\
\text { concentration } \\
C_{e}, \%\end{array}$ \\
\hline $12 \mathrm{kDa}$ & 11.53 & $\sim 1.5$ & No data \\
$22 \mathrm{kDa}$ & 21.9 & $\sim 1.6$ & No data \\
$80 \mathrm{kDa}$ & 79.9 & $\sim 1.8$ & $\sim 9.2$ \\
$400 \mathrm{kDa}$ & 367 & $\sim 2.6$ & $\sim 2.5$ \\
$\mathrm{US}^{\mathrm{a}}$ & No data & No data & $\sim 1.6$ \\
$5 \mathrm{MDa}$ & $5 \times 10^{3}$ & No data & $\sim 1$ \\
\hline
\end{tabular}

${ }^{\mathrm{a}}$ The sample denoted as US was obtained from a $5 \%$ PAA $5 \mathrm{MDa}$ solution in water by ultrasound treatment using a horn sonicator Microson XL-2000 instrument from Misonix Inc. (Farmingdale, NY). The treatment was performed for 10 minutes at a power of $10 \mathrm{~W}$ in a $15 \mathrm{~mL}$ Falcon tube on ice. The protocol included temperature control to prevent heating above $40^{\circ} \mathrm{C}$.

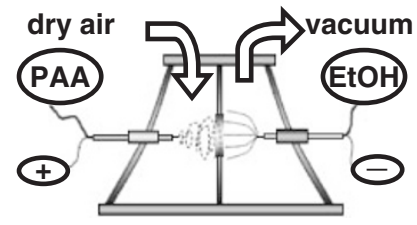

Figure 1. Schematic of the device used to manufacture PAA electrospun nanofibers.

$$
\eta_{\mathrm{sp}}=\left(\eta_{\mathrm{s}}-\eta_{0}\right) / \eta_{0}
$$

where $\eta_{\mathrm{s}}$ and $\eta_{0}$ are zero shear viscosities of solution and solvent, respectively. To find $C_{e}$ we generated a logarithmic plot of $\eta_{\mathrm{sp}} v s$. PAA concentration.

Electrospinning (ES) was conducted using a previously described device ${ }^{33}$ that is schematically shown in Figure 1. In conventional ES techniques charged nano-fibers are generated and then deposited onto a conductive substrate where they are neutralized by giving electrons to or accepting electrons from the substrate. In the present device nanofibers are neutralized on a non-conductive grid or dielectric substrate with a cloud of small counter-ions generated by electrospraying a pure volatile solvent. The following ES conditions were used in all experiments: distance between the substrate and the neutralizer (cathode) $-12 \mathrm{~cm}$, distance between the substrate and spinneret (anode) $-15 \mathrm{~cm}$, voltage between neutralizer and spinneret $-18.2 \mathrm{kV}$, current $-0.45 \pm 0.1 \mu \mathrm{A}$, flow rate of polymer solution $-2 \mu \mathrm{l} / \mathrm{min}$, humidity in the chamber $22 \pm 2 \%$, for neutralization $100 \%$ ethanol was used. Electrospun nanofibers were collected on pre-cleaned Fisherbrand plain microscope slide Fisher Scientific Corp. (Pittsburg, PA) glass plates for AFM and optical microscopy analysis. Simultaneously nanofibers were collected on a polyester mesh (CMY-0105, obtained from Small Parts, Inc., Miami, FL) for optical microscopy. Deposition was performed until visible opaque spot appeared.

Optical microscopy was conducted using an Axiovert-25 Carl Zeiss instrument, GmbH (Jena, Germany) with 40X objective. Images were captured with a Pixera camera (Pixera Corp., Los Gatos, CA) and analyzed by ACD software (ACD System, Ltd, Saanichton, Canada).

\section{Atomic force microscopy}

A Nano-R AFM from Pacific Nanotechnology, Inc (Santa Clara, CA) was used to obtain images of nanofibers in tapping mode. Imaging was performed in air that was dried over silica gel. Fiber height analysis was performed using Femtoscan Online software v. 2.2.71 (Moscow State University \& Advanced Technologies Center, Moscow, Russia).

\section{RESULTS AND DISCUSSION}

In the first series of experiments the viscosity of PAA solutions was measured as a function of concentration and $M_{\mathrm{w}}$. One can see in Figure 2 that the concentration dependence of the specific viscosity could be approximated with a number of connected linear sections with increasing slope. The $C_{e}$ was determined as described in ref $7-11,14,15$ as the concentration at which sections with slopes $\approx 1$ and $>2$ intersect (denoted by arrows in Figure 2). Table I summarizes the

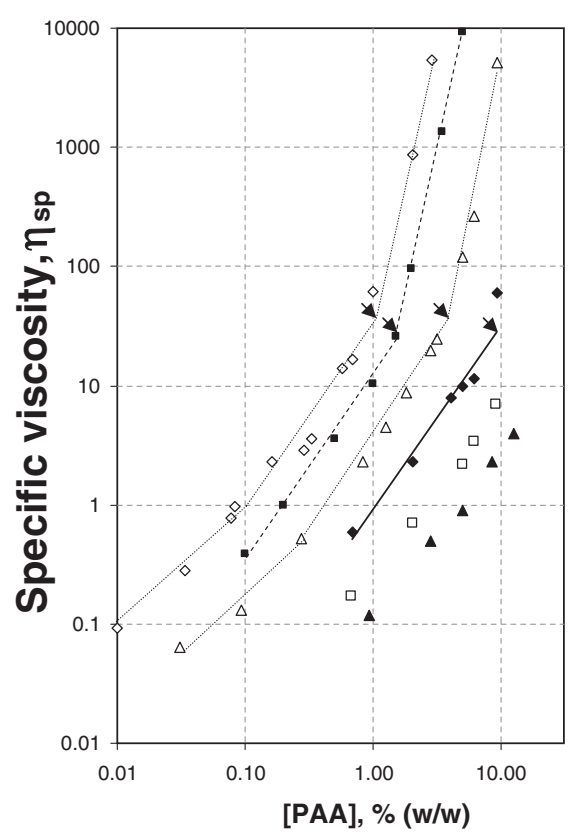

Figure 2. Dependence of specific viscosity, $\eta_{\mathrm{sp}}$, on the weight $\%$ PAA concentration for $\diamond$-PAA-5MDa, 口-PAAUS, $\triangle$-PAA $400 \mathrm{kDa}, \bullet$-PAA $80 \mathrm{kDa}, \square$-PAA $22 \mathrm{kDa}$ and $\boldsymbol{\Delta}$-PAA $12 \mathrm{kDa}$. Arrows denote $C_{e}$ for each polymer. 


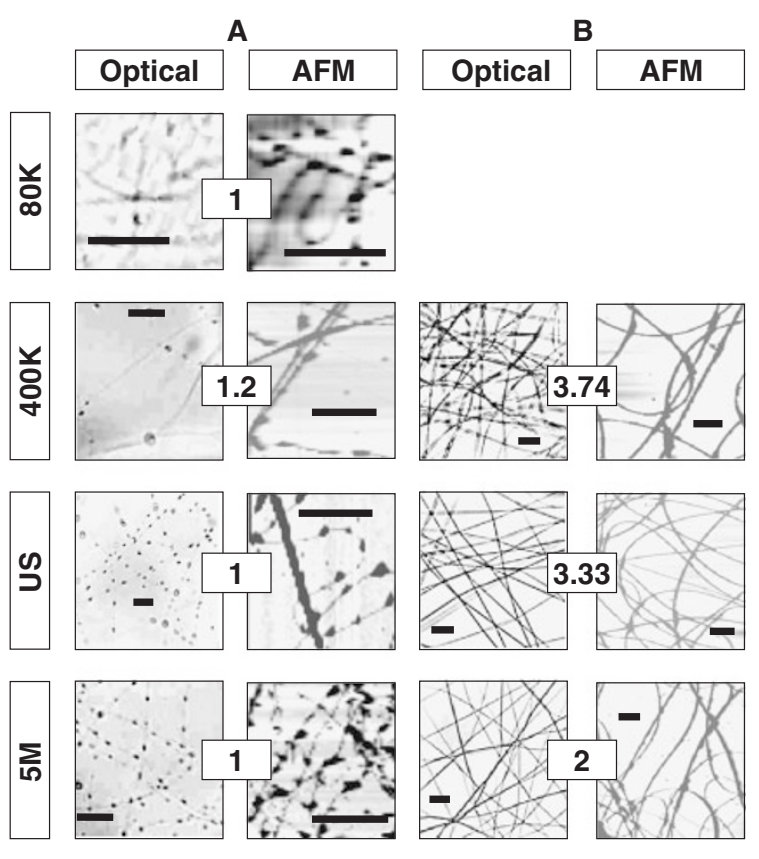

Figure 3. Dependence of nanofibers morphology from $C_{e}$. PAAs of different $M_{\mathrm{w}}$ indicated in boxes on the left side of the picture were electrospun from solutions of different concentration. The method of observation (optical or AFM) is shown in the boxes at the top of the figure while the concentration of the solution normalized to $C_{e}$ is shown in the insert, linking optical and AFM images belonging to the same sample. A - morphology of nanofibers at the concentration $\sim C_{e}$. B - morphology of nanofibers produced at the highest studied concentration. The length of the bar is $10 \mu \mathrm{m}$.

values of $C_{e}$ obtained for each PAA sample studied. The same PAA solutions were then used for electrospinning. PAA solutions of $12 \mathrm{kDa}$ and $22 \mathrm{kDa}$ formed only globular products in these experiments, no fiber formation was observed. The absence of fibers among the ES products can be explained by the fact that all concentrations of these two polymers were below $C_{e}$. PAA with a $M_{\mathrm{w}}$ above $80 \mathrm{kDa}$ produced mostly beads upon electrospraying when $C<C_{e}$ and fibers when $C>C_{e}$, in good agreement with data obtained using other polymers. ${ }^{6-11,14,15}$ Paired images are presented in Figure 3 to illustrate the quality of fibers electrospun at the concentration $C \approx C_{e}$ and at the highest concentration studied for each $M_{\mathrm{w}}$. Beads are only observed at low $C$. This is in good accordance with published data, which show that at polymer concentrations exceeding $C_{e}$ the number of nonfibrous features among electrospinning products decreases dramatically. ${ }^{6-11,14,15}$ As illustrated in Figure 4, the diameter of electrospun PAA fibers, $d$, is linearly dependent on the PAA concentration normalized to $C_{e}, d=\alpha\left(C / C_{e}\right)^{\beta}\left(\alpha=0.11 \mu \mathrm{m} ; \beta=1, \mathrm{R}^{2}=0.9\right)$. Similar empirical formulas with $\beta$ varying from 1.2 to 3.1 and $\alpha$ varying from 0.002 to 0.18 were reported for other polymer-solvent pairs. ${ }^{6-11}$ It is important to

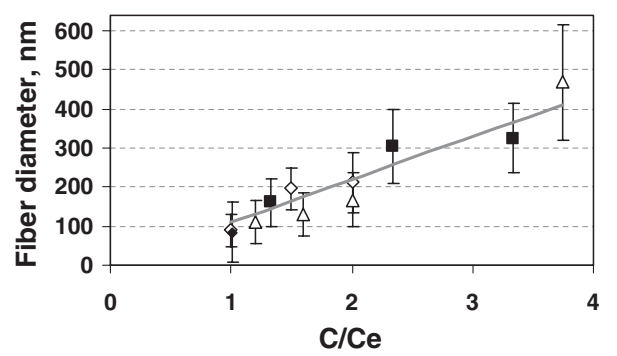

Figure 4. Dependence of the diameter of electrospun fibers on the concentration of the PAA solution normalized to $C_{e}$. $\diamond$-PAA $5 \mathrm{MDa}$, 口-PAAUS, $\triangle$-PAA $400 \mathrm{kDa}, \diamond$-PAA $80 \mathrm{kDa}$.

note that directly plotting $d$ against $C$ does not yield a linear dependence for PAAs of different $M_{w}$, a phenomenon also observed for other polymers. ${ }^{7,10}$ Our results indicate that the empirical relationship between the diameter of electrospun fibers and the normalized polymer concentrations previously observed for a variety of other polymer-solvent systems ${ }^{6-11}$ can also be used to describe electrospinning of aqueous PAA solutions. In the absence of information concerning average $M_{\mathrm{w}}$ and $M_{\mathrm{w}}$ distribution one can predict the outcome of ES by measuring the dependence of the specific viscosity on weight concentration for this polymer and estimating $C_{e}$. Our results with PAA-5MDa solutions treated with ultrasound clearly demonstrate this.

\section{CONCLUSIONS}

We showed that the dependence of viscosity on $M_{\mathrm{w}}$ and PAA concentration normalized to $C_{e}$ allows one to predict the electrospinability of PAA solutions, the quality of nanofibers and their diameter.

Acknowledgment. The authors gratefully acknowledge support from DOE grant, DE-F C5204NA25455. We also acknowledge the valuable advice of Dr. Timothy Born concerning the manuscript.

\section{REFERENCES}

1. D. H. Reneker and I. Chun, Nanotechnology, 7, 216 (1996).

2. T. Subbiah, G. S. Bhat, R. W. Tock, S. Pararneswaran, and S. S. Ramkumar, J. Appl. Polym. Sci., 96, 557 (2005).

3. D. Li and Y. N. Xia, Adv. Mater., 16, 1151 (2004).

4. Z. M. Huang, Y. Z. Zhang, M. Kotaki, and S. Ramakrishna, Compos. Sci. Technol., 63, 2223 (2003).

5. P. G. De Gennes, "Scaling Concepts in Polymer Physics". Cornell University Press, Ithaca, N. Y., 1979.

6. M. G. McKee, C. L. Elkins, and T. E. Long, Polymer, 45, 8705 (2004).

7. M. G. McKee, M. T. Hunley, J. M. Layman, and T. E. Long, Macromolecules, 39, 575 (2006). 
8. M. T. Hunley, M. G. McKee, and T. E. Long, J. Mater. Chem., 228, 605 (2007).

9. M. G. McKee, G. L. Wilkes, R. H. Colby, and T. E. Long, Macromolecules, 37, 1760 (2004).

10. P. Gupta, C. Elkins, T. E. Long, and G. L. Wilkes, Polymer, 46, 4799 (2005).

11. K. Ohkawa, K. I. Minato, G. Kumagai, S. Hayashi, and H. Yamamoto, Biomacromolecules, 7, 3291 (2006).

12. S. L. Shenoy, W. D. Bates, H. L. Frisch, and G. E. Wnek, Polymer, 46, 3372 (2005).

13. S. L. Shenoy, W. D. Bates, and G. E. Wnek, Polymer, 46, 8990 (2005).

14. S. H. Tan, R. Inai, M. Kotaki, and S. Ramakrishna, Polymer, 46, 6128 (2005).

15. C. Wang, C. H. Hsu, and J. H. Lin, Macromolecules, 39, 7662 (2006).

16. S. H. Kim, S. Nair, and E. Moore, Macromolecules, 38, 3719 (2005).

17. V. N. Morozov, T. Y. Morozova, and N. R. Kallenbach, Int. J. Mass Spectrom., 178, 143 (1998).

18. L. Wannatong, A. Sirivat, and P. Supaphol, Polym. Int., 53, 1851 (2004).

19. C. Bruce and W. H. Schwarz, J. Polym. Sci., Polym. Phys. Ed., 7, 909 (1969).

20. J. Klein and K. D. Conrad, Macromol. Chem. Phys., 181,
227 (1980).

21. P. Munk, T. M. Aminabhavi, P. Williams, D. E. Hoffman, and M. Chmelir, Macromolecules, 13, 871 (1980).

22. N. Narkis and M. Rebhun, Polymer, 7, 507 (1966).

23. H. R. Maurer "Disc Electrophoresis and Related Techniques of Polyacrylamide Gel Electrophoresis”. Walter de Gruyter, Berlin, 1971.

24. J. N. Hansen, Anal Biochem., 76, 37 (1976).

25. E. Emilitri, E. Ranucci, and P. Ferruti, J. Polym. Sci., Part A: Polym. Chem., 43, 1404 (2005).

26. E. Karadag, D. Saraydin, and O. Guven, Turk. J. Chem., 21, 151 (1997).

27. E. Karadag, O. B. Uzum, and D. Saraydin, Mater. Des., 26, 265 (2005).

28. E. Karadag, O. B. Uzum, D. Saraydin, and O. Guven, Mater. Des., 27, 576 (2006).

29. D. Saraydin, E. Karadag, and O. Guven, Polym. Adv. Technol., 6, 719 (1995).

30. J. M. Lim, J. H. Moon, G. R. Yi, C. J. Heo, and S. M. Yang, Langmuir, 22, 3445 (2006).

31. H. Hiratani, C. Alvarez-Lorenzo, J. Chuang, O. Guney, A. Y. Grosberg, and T. Tanaka, Langmuir, 17, 4431 (2001).

32. A. Rudin and K. K. Chee, Macromolecules, 6, 613 (1973).

33. V. N. Morozov and N. N. Vsevolodov, Adv. Mater., (2007), submitted. 